\title{
Criminologie
}

\section{Document : Premier rapport annuel du bureau des inspecteurs de prisons, asiles, etc. de la province de Québec pour les années 1867 et 1868}

Volume 18, numéro 1, 1985

L’histoire du contrôle social

URI : https://id.erudit.org/iderudit/017208ar

DOI : https://doi.org/10.7202/017208ar

Aller au sommaire du numéro

Éditeur(s)

Les Presses de l'Université de Montréal

ISSN

0316-0041 (imprimé)

1492-1367 (numérique)

Découvrir la revue

Citer ce document

(1985). Document : Premier rapport annuel du bureau des inspecteurs de prisons, asiles, etc. de la province de Québec pour les années 1867 et 1868. Criminologie, 18(1), 68-71. https://doi.org/10.7202/017208ar d'utilisation que vous pouvez consulter en ligne. 


\section{PREMIER RAPPORT ANNUEL}

DU

BUREAU DES INSPECTEURS DE PRISONS, ASILES, ETC.

DE LA PROVINCE DE QUÉBEC

POUR LES ANNÉES $1867 \& 1868$.

DOCUMENTS DE LA SESSION, 1869, $\mathrm{N}^{\circ} 23$

(EXTRAITS)

À Sir NARCISSE FORTUNAT BELLEAU, Chevalier, LieutenantGouverneur de la Province de Québec.

Qu'il plaise à Votre Excellence : (...)

\section{ARTICLE PREMIER}

Ce qu'étaient nos institutions pénales et de bienfaisance lors de l'établissement d'un bureau d'inspection en 1858.

N'étant alors chargés ni par état ni par devoir de les surveiller, nous ne pouvons en parler qu'en nous servant du langage de ceux qui en avaient alors la direction. C'est ce que nous allons faire, et nous le croyons, avec avantage pour le public. Car au nombre des inspecteurs d'alors, il y avait des observateurs distingués, des médecins habiles, de bons écrivains. Ils vont nous révéler un ensemble de faits d'un caractère tellement déplorable que la société en sera surprise. Et pourtant, personne n'osera douter des paroles d'un homme de mérite et de la position du Docteur J.C. Taché, ni des avancés d'un vieillard aussi souvent honoré de la confiance publique que l'a été le Docteur W. Nelson, ni non plus de la haute honorabilité de leurs collègues, MM. MacDonald, Langton et Meredith. Parlant des Prisons du Haut et du Bas-Canada, voici ce qu'ils en disent dans leur Rapport de 1859 :

«Les Défauts de nos Prisons sont de tous les genres et bien qu'il y ait divers degrés, il n'en reste pas moins vrai qu'aucun de ces établissements ne répond au triple but qu'on doit se proposer en les formant, savoir : punir, contenir et amender. 
Défauts dans la surveillance, défauts dans la discipline, défauts dans les constructions et la distribution intérieure et extérieure des édifices. Défauts dans les dispositions hygiéniques; défauts surtout dans les moyens de moralisation; défauts partout.

Dans la plupart des Prisons, et particulièrement dans celles qui reçoivent habituellement un nombre considérable de détenus, la surveillance est nécessairement nulle, par le fait de l'insuffisance du personnel attaché à chaque prison. Six prisons n'ont qu'un seul individu, le geôlier pour tout faire, et les autres n'ont que deux officiers, le geôlier et un guichetier. Cependant, quelques-unes de ces prisons ont eu jusqu'à quarante à cinquante prisonniers à la fois. Il est évident qu'avec la meilleure volonté du monde, les gardiens de ces maisons ne peuvent en aucune façon exercer de surveillance; leur autorité n'existe de fait que durant bon plaisir des prisonniers.

Les prisons ont été négligées à ce point que dans presque toutes, il n'existe aucune espèce de règlement; les choses vont comme elles peuvent, et le malheureux geôlier est forcé d'en prendre son parti et de se tirer d'affaire sous l'autorité du Shérif qui n'est guère mieux situé que lui sous ce rapport.

Grand nombre de bâtiments employés comme prisons donnent directement sur les chemins, et offrent aux prisonniers des moyens faciles de communiquer avec l'extérieur.

Les Chambres et ce qu'on appelle les cellules dans les prisons actuelles, sont mal disposées et mal établies; le tout ensemble, loin de se prêter à la classification des prisonniers, est, dans la plupart des cas, un obstacle insurmontable à toute tentative de séparation.

Les prisonniers demeurent ensemble pendant le jour, et couchent ensemble (à peu d'exceptions près) par deux, par trois et par six pendant la nuit. En dehors de la séparation plus ou moins parfaite des sexes, on peut dire que les âges, les conditions, les moralités forment dans ces établissements, un pêle-mêle déplorable au milieu duquel nous apparaissent de pauvres aliénés, de malheureux indigents, sans feu ni lieu, et de plus malheureuses créatures encore, qu'une première faute, souvent comparativement légère, condamne presque infailliblement de cette sorte à une perte complète.

Avec le système actuel de nos prisons, qui n'est absolument qu'une absence complète de tout système, on manque tout à fait le but des Institutions pénales : on ne punit pas, ou on punit mal, on ne détourne point du crime et on n'améliore point le condamné. 
La plupart des habitués de nos Prisons ne sont nullement punis par leur séjour temporaire dans ces maisons qui pour une certaine classe de délinquants ne sont qu'un Asile pour le besoin des mauvais jours.

Là ils sont traités gratuitement pour les maladies contractées par l'abus des liqueurs et la débauche; là ils rencontrent librement d'anciens ou de nouveaux amis, là ils complotent contre la société, et organisent leurs prochaines campagnes, en enrôlant dans leur triste bande de nouvelles recrues.

$\mathrm{Si}$, pour quelques-uns, la Prison actuellement est une punition, alors il n'y a aucune proportion dans la peine, et en dépit de la lettre de la loi, et de la sentence du juge, le degré de châtiment est laissé aux chances du lieu où sera exécutée telle sentence. En effet, dans telle prison, la discipline est à peu près quelque chose, dans telle autre, elle est nulle; dans telle prison, le logement est affreux, dans telle autre, il est passable; dans telle prison on travaille, dans telle autre, les prisonniers ne font rien, pas même ceux que le tribunal a condamnés aux travaux forcés. Dans certaines prisons, on punit les infractions à la discipline, dans d'autres, il n'y a ni discipline ni punition.

On peut juger par tout ce qui précède, que la Législation et l'hygiène ne sont guère appelées chez nous à traiter les passions de ceux qui ont été conduits par elles à nuire à la société. Rien n'a été fait pour pourvoir aux besoins religieux des prisonniers, et cependant sans la religion, il n'est, pour personne, de moralisation possible sur la terre.

Il est un détail lamentable qu'il ne faut pas cacher et que tous doivent connaître, parce qu'il constitue un mal auquel les individus, les famille, et l'État sont tenus d'apporter remède : On entend parler du rôle que joue la prostitution dans ce pénible état de chose.

Presque toutes les Prisonnières sont des Prostituées pour qui nos Prisons servent de pensionnat et de retraite... ...Il faut se hâter de le dire à la suite de beaucoup d'officiers, d'aumôniers et de médecins de nos Institutions pénales, nos Prisons communes sont des écoles de vice où dans le sein de l'indolence et des voluptés de la crapule, les jeunes délinquants vont apprendre les roueries du mal, de ceux qui, plus vieux dans le crime, s'en constituent les précepteurs et les modèles.

D'après ce qui précède, il est facile de se convaincre que la tâche imposée à nos prédécesseurs, était lourde, et qu'il leur a fallu un long et pénible travail pour indiquer les remèdes à un mal si profond.

Malgré toute leur bonne volonté, malgré tous leurs efforts, et malgré leurs hautes capacités, ils ont été eux-mêmes forcés d'avouer, dans 
quelques-uns de leurs rapports, qu'ils n'avaient pas été assez heureux pour parvenir à donner une solution définitive aux graves et difficiles problèmes qu'ils avaient à résoudre, ni pour faire disparaître tout le mal qu'ils avaient dépeint sous d'aussi sombres couleurs, et pour la guérison duquel ils avaient indiqué bien des remèdes.

Une des principales causes qui a dû entraver, sinon paralyser leurs efforts, c'est que le pays n'a pas jugé à propos de faire les sacrifices exigés pour mettre à effet tous leurs plans et toutes leurs suggestions. Il en sera de même pour nous, si la Législature ne vient pas à notre aide, en votant les subsides nécessaires à la construction, à l'agrandissement et à la réparation de nos prisons, etc. Nous avons raison de croire qu'il en sera autrement et que d'année en année, sans trop surcharger le budget, la Législature fera un octroi généreux pour les fins mentionnées dans le présent rapport.

D'ailleurs, ces dépenses seront amplement remboursées par la diminution des déprédations que commettent les filous et les voleurs dans le pays, sans tenir compte de la protection que retire la société de la bonne administration de ces Institutions ni de la Réforme morale et religieuse des prisonniers. 